\title{
A Poset Classifying Non-Commutative Term Orders
}

\author{
Jan Snellman \\ Department of Mathematics Linköping University SE-58183 Linköping Sweden jasne@mai. Iiu.se
}

received February 4, 2001, revised April 16, 2001, accepted April 20, 2001.

\begin{abstract}
We study a poset $\mathfrak{N}$ on the free monoid $X^{*}$ on a countable alphabet $X$. This poset is determined by the fact that its total extensions are precisely the standard term orders on $X^{*}$. We also investigate the poset classifying degree-compatible standard term orders, and the poset classifying sorted term orders. For the latter poset, we give a Galois coconnection with the Young lattice.
\end{abstract}

Keywords: free associative algebra, term orders

\section{Introduction}

So-called strongly stable ideals are much studied in commutative algebra because of their intimate connection with generic initial ideals $[7,8,5]$, because their rôle in elucidating Macaulays theorem on possible Hilbert functions [3, 2, 4] and because their minimal free resolutions have a simple structure [6]. In brief, a monomial ideal $I$ in a polynomial ring $\mathbb{C}\left[x_{1}, \ldots, x_{n}\right]$ is strongly stable ${ }^{\dagger}$ if, whenever $m=x_{1}^{a_{1}} \cdots x_{n}^{a_{n}} \in I$, then $\frac{x_{j+1}}{x_{j}} m \in I$ for all $j$ such that $j<n, a_{j}>0$. So, we should be able to replace any occurring $x_{j}$ with $x_{j+1}$, as long as $x_{j+1} \in\left\{x_{1}, \ldots, x_{n}\right\}$.

Now let $V$ denote the vector space of linear forms in $\mathbb{C}\left[x_{1}, \ldots, x_{n}\right]$, and let $G_{n}$ denote the general linear group on $V$. Then $G_{n}$ acts on $V$, and also on $\mathbb{C}\left[x_{1}, \ldots, x_{n}\right] \simeq S(V)$, the symmetric algebra on $V$. This action is by linear substitution of variables. The ideals fixed by the subgroup of $G_{n}$ consisting of the diagonal matrices are precisely the monomial ideals, and the ideals fixed by the subgroup consisting of the upper triangular matrices are precisely the strongly stable monomial ideals.

Since a monomial ideal in $\mathbb{C}\left[x_{1}, \ldots, x_{n}\right]$ correspond to a monoid ideal in $\left[x_{1}, \ldots, x_{n}\right]$, the free abelian monoid on $\left\{x_{1}, \ldots, x_{n}\right\}$, and since monoid ideals corresponds to filters in $\left(\left[x_{1}, \ldots, x_{n}\right], D\right)$, where $D$ denotes the divisibility partial order, it is natural to ask the question: is there a partial order $\mathfrak{C}_{n}$ on $\left[x_{1}, \ldots, x_{n}\right]$, such that its filters are precisely the strongly stable monomial ideals? And if so, what are its properties?

It is clear that there is such a poset: simply define it as the smallest poset containing all relations $m \leq \mathrm{tm}$ and $m \leq \frac{x_{i+1}}{x_{i}} m$. What is more interesting are the following two results, proved in [12]:

\footnotetext{
$\dagger$ In the literature, it is more common to insist on the reverse order of the variables, thus $x_{j}$ is replaced with $x_{j-1}$. For our purposes (particularly since we will be dealing with infinitely many variables) our definition is more convenient.
} 


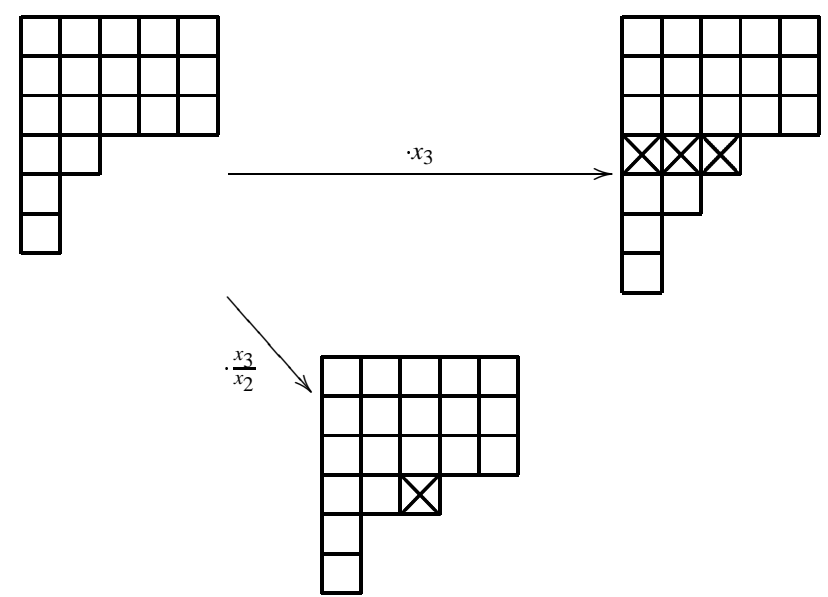

Fig. 1: $x_{1}^{2} x_{2} x_{5}^{3} \cdot x_{3}$ and $x_{1}^{2} x_{2} x_{5}^{3} \cdot \frac{x_{3}}{x_{2}}$

A) Define a standard term order on $\left[x_{1}, \ldots, x_{n}\right]$ to be a total order $>$ such that $1<x_{1}<\cdots<x_{n}$, and such that $m_{1}<m_{2} \Longrightarrow t m_{1}<t m_{2}$. Then $\mathfrak{C}_{n}$ is the intersection of all standard term orders.

B) Define a map from $\left[x_{1}, \ldots, x_{n}\right]$ to the set of Ferrers diagrams with at most $n$ columns, as follows: $x_{1}^{a_{1}} \cdots x_{n} a_{n}$ goes to the diagram with $a_{i}$ rows of length $i$, for $1 \leq i \leq n$. Then multiplication with $x_{j}$ corresponds to inserting an extra row of length $j$, and multiplication with $\frac{x_{j+1}}{x_{j}}$ corresponds to removing one row of length $j$ and inserting one row of length $j+1$, i.e. to the insertion of an extra box (this is illustrated in Figure 1). Thus, the map is an isotone bijection with isotone inverse, showing that $\mathfrak{C}_{n}$ is isomorphic to a sub-lattice of the Young lattice.

Since the poset $\mathfrak{C}_{n}$ is canonically embedded in $\mathfrak{C}_{n+1}$, we can let $n$ tend to infinity and study what happens when we have countably many indeterminates. It is a pleasing but unsurprising fact that the poset $\mathfrak{C}=\mathfrak{C}_{\infty}$ that we obtain in this way is isomorphic to the Young lattice.

In this article, we study the non-commutative analogue $\mathfrak{N}_{n}$ of the poset $\mathfrak{C}_{n}$. We show that this poset bears the same relation to non-commutative term orders, and to non-commutative monomial ideals fixed by upper triangular matrices, as does its commutative counterpart. However, $\mathfrak{N}_{n}$ is not a lattice, as we can see from Figure 2 on page 305, the Hasse diagram of $\mathfrak{N}_{2}$.

Our motivation for studying $\mathfrak{N}_{n}$ is on one hand its relation to "non-commutative generic initial ideals", and on the other hand its relation to term orders. We believe that it can be used to determine the possible order types: recall the result of Martin and Scott [10] that the possible order types for term orders on the free monoid on two letters are $\omega, \omega^{2}$ and $\omega^{\omega}$.

We also believe that $\mathfrak{N}_{n}$ is of interest "in itself"; we plan, in a subsequent article, to give an account of its incidence algebra.

It is possible to define partial orders which capture the properties of generic initial ideals over fields of finite characteristic. Already in the commutative case, these partial orders are immensely complicated; 
they involve the so-called "Gauss order" on the integers in a non-trivial way (see the PhD thesis of Keith Pardue[11] for more details.) Their non-commutative counterparts should be even more formidable; we will avoid these added complications and deal exclusively with the characteristic zero case.

\section{Notations}

We will use the terminology of [1] for partially ordered sets. Let $n$ be a positive integer, let $X=$ $\left\{x_{1}, x_{2}, x_{3}, \ldots\right\}$ be a denumerable set of indeterminates, and let $X_{n}=\left\{x_{1}, \ldots, x_{n}\right\}$. Let $X^{*}, X_{n}^{*}$ be the corresponding free (non-abelian) monoid, and $[X],\left[X_{n}\right]$ be the corresponding free abelian monoids. We let $D$ denote the divisibility partial order on $X^{*}$ and on $[X]$ (and, by restriction, on $X_{n}^{*}$ and on $\left[X_{n}\right]$ ).

Definition 2.1. We define

$$
\begin{gathered}
\sigma: X^{*} \rightarrow[X] \\
m=x_{i_{1}} \cdots x_{i_{d}} \mapsto x_{1}^{a_{1}} \cdots x_{N}^{a_{N}}
\end{gathered}
$$

where $N$ is the highest index of a variable occurring in $m$, and $a_{j}$ denotes the number of occurrences of $x_{j}$ in $m$.

We also define

$$
\begin{aligned}
\sigma^{+}:([X], \mathfrak{C}) & \rightarrow\left(X^{*}, \mathfrak{N}\right) \\
x_{1}^{a_{1}} \cdots x_{\ell}^{a_{\ell}} & \mapsto x_{1}^{a_{1}} \cdots x_{\ell}^{a_{\ell}}
\end{aligned}
$$

Lemma 2.2. $\sigma$ and $\sigma^{+}$are order-preserving with respect to the divisibility partial order on $X^{*}$ and $[X]$. For $m \in X^{*}, t \in[X]$, we have that $\sigma\left(\sigma^{+}(t)\right)=t$ and that $\sigma^{+}(\sigma(m))$ is the "sorted version" of $m$; if $m=x_{i_{1}} \cdots x_{i_{d}}$ then $\sigma^{+}(\sigma(m))=x_{i_{\tau(1)}} \cdots x_{i_{\tau(d)}}$ for some permutation $\tau$ of $\{1, \ldots, d\}$.

Proof. Obvious.

Let $\mathbb{N}^{\omega}$ be the subset of $\mathbb{N}^{\left(\mathbb{N}^{+}\right)}$consisting of finitely supported sequences, so $\mathbb{N}^{\omega}$, with the natural order relation, is order isomorphic to $([X], D)$. We denote this isomorphism by exp, and its inverse by log. We put $\mathbf{e}_{i}=(0, \ldots, 0,1,0 \ldots)$, where the single 1 is in the $i$ ' th position.

We let $\Sigma$ be the functional which assigns to each element in $\mathbb{N}^{\omega}$ the sum of its components, and we let $S$ be the (left) shift operator. The $i$ ' th projection map $\mathbb{N}^{\omega} \rightarrow \mathbb{N}$ is denoted by $\pi_{i}$.

Definition 2.3. Let $M$ be a monoid. A partial order on $M$ is multiplicative (is a monoid partial order) if

- $1<t$ for all $t \in M \backslash\{1\}$,

- If $s<t$ then $a s b<a t b$ for all $a, b \in M$.

Definition 2.4. A multiplicative total order $<$ on $X^{*}$ (or on $X_{n}^{*}$ ) is called a term order. A term order is standard if

$$
x_{1}<x_{2}<x_{3}<\cdots
$$

A result by Higman [9] implies that a standard term order is a well order. 


\section{Definition and basic properties of $\mathfrak{N}$}

Definition 3.1. Regarding a term order on $X^{*}$ as a subset of $X^{*} \times X^{*}$, we define the partial order $\mathfrak{N}$ to be the intersection of all standard term orders on $X^{*}$. We define $\mathfrak{N}_{n}$ to be the intersection of all standard term orders on $X_{n}^{*}$.

Lemma 3.2. $\mathfrak{N}_{n}$ is the restriction of $\mathfrak{N}$.

Proof. Every standard term order on $X^{*}$ restricts to a standard term order on $X_{n}^{*}$, and every standard term order on $X_{n}^{*}$ can be extended to a standard term order on $X^{*}$ : just take the lexicographic product with some standard term order on $\left(X \backslash X_{n}\right)^{*}$.

Proposition 3.3. $\mathfrak{N}$ and $\mathfrak{N}_{n}$ are locally finite well partial orders whose principal order ideals are finite.

Proof. Let $m \in X^{*}$. Since $\mathfrak{N}$ is the intersection of all standard term orders, we have that if we pick one such standard term order, $>$, then the principal order ideal on $m$ with respect to $\mathfrak{N}$ is contained in the principal order ideal on $m$ with respect to $>$. Since $>$ is a well total order, this latter set is finite.

Any poset whose principal order ideals are finite is a locally finite well partial order, so the result follows.

Definition 3.4. For $i \in \mathbb{N}^{+}$, define the $i$ 'th raising operation as the partially defined map

$$
\begin{aligned}
R_{j}: X^{*} & \rightarrow X^{*} \\
m=x_{i_{1}} \cdots x_{i_{d}} & \mapsto x_{i_{1}} \cdots x_{i_{j-1}} x_{i_{j}+1} x_{i_{j+1}} \cdots x_{i_{d}}
\end{aligned}
$$

This is defined for $j \leq d$. As an operation from $X_{n}^{*} \rightarrow X_{n}^{*}, R_{j}\left(x_{i_{1}} \cdots x_{i_{d}}\right)$ is defined when $j \leq d$ and $i_{j}<n$.

Theorem 3.5. (i) The standard term orders on $X^{*}$ correspond to total multiplicative extensions of $\mathfrak{N}$. Similarly, the standard term orders on $X_{n}^{*}$ correspond to total multiplicative extensions of $\mathfrak{N}_{n}$.

(ii) $\mathfrak{N}$ and $\mathfrak{N}_{n}$ are monoid partial orders.

(iii) For $m, m^{\prime} \in X^{*}$, we have that $m \leq m^{\prime}$ with respect to $\mathfrak{N}$ if and only if $m^{\prime}$ can be obtained from $m$ by a finite sequence of applications of the following rules:
(a) $t \mapsto x_{1} t$
(b) $t \mapsto t x_{1}$
(c) $t \mapsto R_{j}(t)$.

The corresponding result holds for $\mathfrak{N}_{n}$.

Proof. We prove the results for $\mathfrak{N}$, the ones for $\mathfrak{N}_{n}$ are similar.

(i) If $<$ is a multiplicative total extension of $\mathfrak{N}$, then it is a multiplicative total order on $X^{*}$, hence a term order. Since it extends $\mathfrak{N}$, it follows that $x_{1}<x_{2}<x_{3}<\cdots$ so $<$ is standard. Conversely, if $<$ is a standard term order, then $x_{1}<x_{2}<x_{3}<\cdots$ and $<$ is a multiplicative total order on $X^{*}$. Furthermore, if $m \leq m^{\prime}$ with respect to $\mathfrak{N}$, then $m \leq m^{\prime}$ with respect to all standard term orders, so in particular $m<m^{\prime}$ or $m=m^{\prime}$. Hence $<$ extends $\mathfrak{N}$. 
(ii) Suppose that $m \leq m^{\prime}$ with respect to $\mathfrak{N}$. Then $m \leq m^{\prime}$ with respect to every standard term order, hence $s m t \leq s m^{\prime} t$ with respect to every standard term order, hence $s m t \leq s m^{\prime} t$ with respect to $\mathfrak{N}$.

(iii) If $>$ is a standard term order on $\mathfrak{N}$, then for all $t$,

$$
t<x_{1} t, \quad t<t x_{1}, \quad t \leq R_{j}(t)
$$

Hence if $m^{\prime}$ is obtained from $m$ by a sequence of operations (iiia), (iiib), (iiic), then $m^{\prime} \geq m$. Since this holds for any standard term order, $m^{\prime} \geq m$ with respect to $\mathfrak{N}$.

Conversely, suppose that $m^{\prime} \geq m$ with respect to $\mathfrak{N}$. Then $m^{\prime} \geq m$ with respect to all standard term orders, in particular with respect to the total degree orders. So $\left|m^{\prime}\right| \geq|m|$. Furthermore, it is clear that regarded as a subset of $X^{*} \times X^{*}, \mathfrak{N}$ is the smallest partial order which is also a bi- $\mathfrak{N}$-module containing $\left\{(s m t, m) \mid s, t, m \in X^{*}\right\}$ and $\left\{\left(x_{j}, x_{i}\right) \mid j>i\right\}$, the multiplication being $s(a, b) t=(s a t, s b t)$. We can, in fact, replace these generators by the following: $\left\{(t, 1) \mid t \in X^{*}\right\}$, and $\left\{\left(x_{i+1}, x_{i}\right) \mid i \in \mathbb{N}^{+}\right\}$. But

$$
\begin{aligned}
x_{i+1} & =R_{1}\left(x_{i}\right) \\
x_{i_{1}} \cdots x_{i_{d}} & =R_{d}^{i_{d}} \circ \cdots \circ R_{2}^{i_{2}} \circ R_{1}^{i_{1}}\left(x_{1}^{d}\right) \\
& =R_{d}^{i_{d}} \circ \cdots \circ R_{2}^{i_{2}} \circ R_{1}^{i_{1}}\left(1 \cdot x_{1} \cdots x_{1}\right),
\end{aligned}
$$

so $x_{i+1}$ can be obtained from $x_{i}$ by one application of a raising operator, and $t=x_{i_{1}} \cdots x_{i_{d}}$ can be obtained from 1 by a $d$ right multiplications by $x_{1}$, followed by an appropriate sequence of raising operators.

Note that $\mathfrak{N}$ and $\mathfrak{N}_{n}$ have non-multiplicative total extensions as well. As an example, if we start extending $\mathfrak{N}_{2}$ by first removing the anti-chain $\left\{x_{1}^{2}, x_{2}\right\}$ by declaring that $x_{2}>x_{1}^{2}$, then in order to have a multiplicative total extension, we must insist that $x_{1} x_{2}>x_{1}^{3}, x_{2} x_{1}>x_{1}^{3}, x_{2}^{2}>x_{1}^{2} x_{2}$, et cetera, and not the other way around. A non-multiplicative total extension may order these anti-chains independently.

Lemma 3.6. Let $m=x_{i_{1}} \cdots x_{i_{d}}, N=\max \left(\left\{i_{1}, \ldots, i_{d}\right\}\right)$. Let $a_{i}$ denote the number of occurrences of $x_{i}$ in $m$; in other words, $\left(a_{1}, a_{2}, a_{3}, \ldots\right)=\log (\sigma(m))$. Then

(i) $m$ is covered by the following words in $X^{*}$ :

- $x_{1} m$ and $m x_{1}$,

- The $a_{1}$ words obtained by replacing one occurrence of $x_{1}$ by $x_{2}$, the $a_{2}$ words obtained by replacing one occurrence of $x_{2}$ by $x_{3}$, and so on, up to and including the $a_{N}$ words obtained by replacing one occurrence of $x_{N}$ by $x_{N+1}$.

If $m \neq x_{1}^{k}$, then these words are distinct, so that $m$ is covered by exactly

$$
2+\Sigma(\log (\sigma(m)))=2+\sum_{i=1}^{N} a_{i}=2+\sum_{j=1}^{d} i_{j}
$$

different words. On the other hand, $x_{1}^{k}$ is covered by $x_{k+1}$, and by the $k$ words $x_{1}^{a} x_{2} x_{1}^{k-a-1}, 0 \leq a \leq$ $k-1$.

(ii) In $X_{n}^{*}$, for $N \leq n$, we have that $m$ is covered by 
- $x_{1} m$ and $m x_{1}$,

- The $a_{1}$ words obtained by replacing one occurrence of $x_{1}$ by $x_{2}$, the $a_{2}$ words obtained by replacing one occurrence of $x_{2}$ by $x_{3}$, and so on, up to and including the $a_{n-1}$ words obtained by replacing one occurrence of $x_{n-1}$ by $x_{n}$.

If $m \neq x_{1}^{k}$, then these words are distinct, so that $m$ is covered by exactly

$$
2+\sum_{i=1}^{n-1} a_{i}
$$

different words.

(iii) The following words are covered by $m$ (both in $X^{*}$ and in $X_{n}^{*}$ ):

- $x_{i_{2}} \cdots x_{i_{d}}$, if $i_{1}=1$,

- $x_{i_{1}} \cdots x_{i_{d-1}}$, if $i_{d}=1$,

- The $a_{2}$ words obtained by replacing on occurrence of $x_{2}$ with $x_{1}$, and so on, up to and including the $a_{N}$ words obtained by replacing one occurrence of $x_{N}$ by $x_{N-1}$.

If $m \neq x_{1}^{k}$, then these words are distinct, so that $m$ covers exactly

$$
b+\sum_{i=2}^{n} a_{i}=b+\Sigma(S(\log (\sigma(m)))), \quad b= \begin{cases}0 & i_{1} \neq 1, i_{d} \neq 1 \\ 1 & i_{1} \neq 1, i_{d}=1 \\ 1 & i_{1}=1, i_{d} \neq 1 \\ 2 & i_{1}=i_{d}=1\end{cases}
$$

different words. $x_{1}^{k}$ covers exactly 1 word, namely $x_{1}^{k-1}$.

Proof. This is immediate from Theorem 3.5 on page 302.

\section{Strongly stable ideals}

Let $V$ be the complex vector space spanned by $X$, and let $G$ be the group of linear automorphisms of $V$. Denote by $T(V)$ the tensor algebra on $V$. Then $X^{*}$ is a basis of $T(V)$ (as a vector space), and $T(V) \simeq$ $\mathbb{C}\left[X^{*}\right]$, the free non-commutative polynomial ring on $X$. Furthermore, the action of $G$ on $V \simeq T(V)_{1}$ extends to an action on all of $T(V)$ : we define

$$
g \cdot x_{i_{1}} \cdots \cdot x_{i_{d}}=\left(g \cdot x_{i_{1}}\right) \cdots\left(g \cdot x_{i_{d}}\right)
$$

and extend this $\mathbb{C}$-linearly.

By analogy with the commutative situation, we make the following definition:

Definition 4.1. The subgroup of upper triangular transformations in $G$ is defined by

$$
U=\left\{u \in G \mid u\left(x_{i}\right)=\sum_{j=i}^{\infty} c_{i j} x_{j} \text { for all } i\right\}
$$




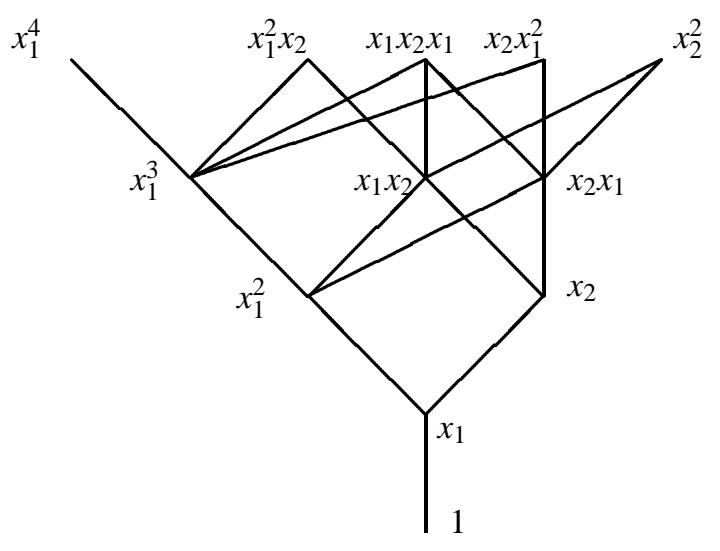

Fig. 2: The Hasse diagram of $\mathfrak{N}_{2}$.

We note that the sums in (6) are finite, and that we must have that $c_{i i} \neq 0$; otherwise, $u$ would not be invertible.

Definition 4.2. A monomial ideal in $\mathbb{C}\left[X^{*}\right]$ is strongly stable if it is fixed under the action of $U$.

Theorem 4.3. The strongly stable monomial ideals in $\mathbb{C}\left[X^{*}\right]$ correspond bijectively to filters in $\mathfrak{N}$.

Proof. Let $I$ be a monomial ideal fixed by $U$. Take any monomial $m \in I, m=x_{a_{1}} \cdots c_{a_{d}}$. Define $u \in U$ by $\left.u\left(x_{a_{1}}\right)\right)=x_{a_{1}}+x_{a_{1}+1}, u\left(x_{j}\right)=x_{j}$ for $j \neq a_{1}$. Then

$$
u(m)=\left(x_{a_{1}}+x_{a_{2}}\right) u\left(x_{a_{2}} \cdots c_{a_{d}}\right)=m+m^{\prime}+m^{\prime \prime}+\cdots,
$$

where $m^{\prime}=R_{1}(m)$, and the rest of the terms are similarly obtained from $m$ by replacing one or several occurrences of $x_{a_{1}}$ by $x_{a_{1}+1}$. All those terms must be in $I$, since $I$ is a monomial ideal. By choosing different $u$ 's, we get that $I$ contains all monomials obtainable from $m$ by means of a single raising operation. Since it is a monomial ideal, it contains also $x_{1} m$ and $m x_{1}$. Hence, from Theorem 3.5 on page 302 we get that the set of monomials in $I$ is a filter with respect to $\mathfrak{N}$.

We get the corresponding result for the case of $n$ variables: we let $V_{n}$ be the vector space spanned by $X_{n}$, then $T\left(V_{n}\right) \simeq \mathbb{C}\left[X_{n}^{*}\right], G_{n}$ is the general linear group on $V$ and can be identified with the set of invertible $n \times n$ matrices, and $U_{n}$ with the set of upper triangular matrices. The $n$ variable version of Theorem 4.3 holds true.

\section{The multi-ranking on $\mathfrak{N}$}

Recall that a locally finite poset $(P, \geq)$ is ranked if there exists a rank function $\Phi: P \rightarrow \mathbb{N}$ such that if $m$ covers $m^{\prime}$ in $P$, then $\Phi(m)=\Phi\left(m^{\prime}\right)+1$. In complete analogy, we define: 
Definition 5.1. A locally finite poset $(P, \geq)$ is said to be $\omega$-multi-ranked if there exists a map

$$
\Phi: P \rightarrow \mathbb{N}^{\omega}
$$

such that

$$
m>m^{\prime} \quad \Longrightarrow \quad \Phi(m)>\Phi\left(m^{\prime}\right)
$$

The poset is $n$-multi-ranked if there exists a map

$$
\Phi_{n}: P \rightarrow \mathbb{N}^{n}
$$

such that

$$
m>m^{\prime} \quad \Longrightarrow \quad \Phi_{n}(m) \gg \Phi_{n}\left(m^{\prime}\right)
$$

Lemma 5.2. Let $P$ be a locally finite poset, and let $1 \leq a \leq b$. Then

$$
P \text { is } \omega \text {-ranked } \Longrightarrow P \text { is b-ranked } \Longrightarrow P \text { is a-ranked } \Longrightarrow P \text { is } 1 \text {-ranked } \Longleftrightarrow P \text { is ranked. }
$$

As an example, we see that the Young lattice is $\omega$-ranked, with the multi-rank-function given by the natural embedding into $\mathbb{N}^{\omega}$ : thus a partition is multi-ranked by the sequence of lengths of the rows in its Ferrers diagram. Collapsing this ranking, we get the ordinary rank function, which ranks an element in the Young lattice by the number of boxes in its Ferrers diagram.

Theorem 5.3. $\mathfrak{N}$ is $\omega$-multi-ranked, and $\mathfrak{N}_{n}$ is n-multi-ranked.

Proof. Let $m=x_{i_{1}} \cdots x_{i_{d}} \in X^{*}$, and put

$$
\mathbf{a}=\log (\sigma(m))=\left(a_{1}, a_{2}, a_{3}, \ldots\right) \in \mathbb{N}^{\omega},
$$

where $a_{j}$ denotes the number of occurrences of $x_{j}$ in $m$. We give $m$ multi-rank in the following way:

$$
\pi_{j}(\Phi(m))=\Sigma\left(S^{j-1}(\mathbf{a})\right)
$$

We can decompose $\Phi=G \circ \log \circ \sigma$, where $G$ is the linear map

$$
\begin{aligned}
G: \mathbb{N}^{\omega} & \rightarrow \mathbb{N}^{\omega} \\
G\left(\mathbf{e}_{i}\right) & =\mathbf{f}_{i}=\sum_{j=1}^{i} \mathbf{e}_{j}
\end{aligned}
$$

Now suppose that $m^{\prime}$ covers $m$. By Lemma 3.6 on page 303, there are two cases:

- $m^{\prime}=x_{1} m$ or $m^{\prime}=m x_{1}$. We see that

$$
\begin{aligned}
\Phi\left(m^{\prime}\right)-\Phi(m) & =\left(G\left(\mathbf{a}+\mathbf{e}_{1}\right)\right)-G(\mathbf{a}) \\
& =\left(G(\mathbf{a})-G\left(\mathbf{e}_{1}\right)\right)-G(\mathbf{a}) \\
& =G\left(\mathbf{e}_{1}\right) \\
& =\mathbf{e}_{1}
\end{aligned}
$$


- $m^{\prime}$ is obtained from $m$ by replacing one occurrence of $x_{j}$ with an $x_{j+1}$. Then

$$
\begin{aligned}
\Phi\left(m^{\prime}\right)-\Phi(m) & =G\left(\mathbf{a}-\mathbf{e}_{j}+e j+1\right)-G(\mathbf{a}) \\
& =-G\left(\mathbf{e}_{j}\right)+G\left(\mathbf{e}_{j+1}\right) \\
& =\mathbf{e}_{j+1}
\end{aligned}
$$

This shows that $\Phi$ is a multi-rank function. The function $\Phi_{n}$ is defined by restriction.

By collapsing the ranking, we get

Theorem 5.4. $X^{*}$ and $X_{n}^{*}$ are ranked posets. The rank of the word $x_{i_{1}} \cdots x_{i_{d}}$, with $a_{j}$ occurrences of the letter $x_{j}$, is $\sum_{j=1}^{\infty} j a_{j}$. The rank multi-generating functions for $X^{*}$ and $X_{n}^{*}$ are, respectively

$$
\frac{1}{1-\sum_{i=1}^{\infty} t^{i} x_{i}}, \quad \text { and } \quad \frac{1}{1-\sum_{i=1}^{n} t^{i} x_{i}},
$$

the rank-generating functions are

$$
\frac{1-t}{1-2 t}, \quad \text { and } \quad \frac{1-t}{1-2 t+t^{n+1}}
$$

Proof. We prove the formulæs for $X^{*}$. The ranking $\Phi$ gives $x_{i}$ weight $(1, \ldots, 1,0, \ldots)$, with $i$ consecutive ones. For the standard weights, i.e. $x_{i}$ has weight $(0, \ldots, 1,0 \ldots)$, with the 1 at position $i$, the generating function is $\frac{1}{1-\sum_{i=1}^{\infty} x_{i}}$. Thus, substituting $\prod_{j=1}^{i} t_{j}$ for $x_{i}$, we obtain the rank-generating function

$$
\frac{1}{1-\sum_{i=1}^{\infty} \prod_{j=1}^{i} t_{j}}
$$

which specialises to

$$
\frac{1}{1-\sum_{i=1}^{\infty} \prod_{j=1}^{i} t}=\frac{1}{1-\sum_{i=1}^{\infty} t^{i}}=\frac{1}{1-t \frac{1}{1-t}}=\frac{1-t}{1-2 t} .
$$

In Figure 3 on the following page, we give the Hasse diagram of $\mathfrak{N}$ up to and including rank level 3.

\section{Relation to commutative term orders}

Definition 6.1. Let $\mathfrak{C}$ denote the smallest partial order on the free abelian monoid $[X]$ such that

1. $1 \leq m$ for all $m \in[X]$,

2. $m \leq m^{\prime} \Longrightarrow t m \leq t m^{\prime}$ for all $m, m^{\prime}, t \in[X]$,

3. $x_{1}^{a_{1}} \cdots x_{n}^{a_{n}} \leq \frac{x_{i+1}}{x_{i}} x_{1}^{a_{1}} \cdots x_{n}^{a_{n}}$ whenever $a_{i}>0$.

For any $n$, denote by $\mathfrak{C}_{n}$ the restriction of this partial order to $\left[X_{n}\right]=\left[\left\{x_{1}, \ldots, x_{n}\right\}\right]$.

The following theorem was proved in [12] (parts 3 and 4 can be easily derived from [11]). 


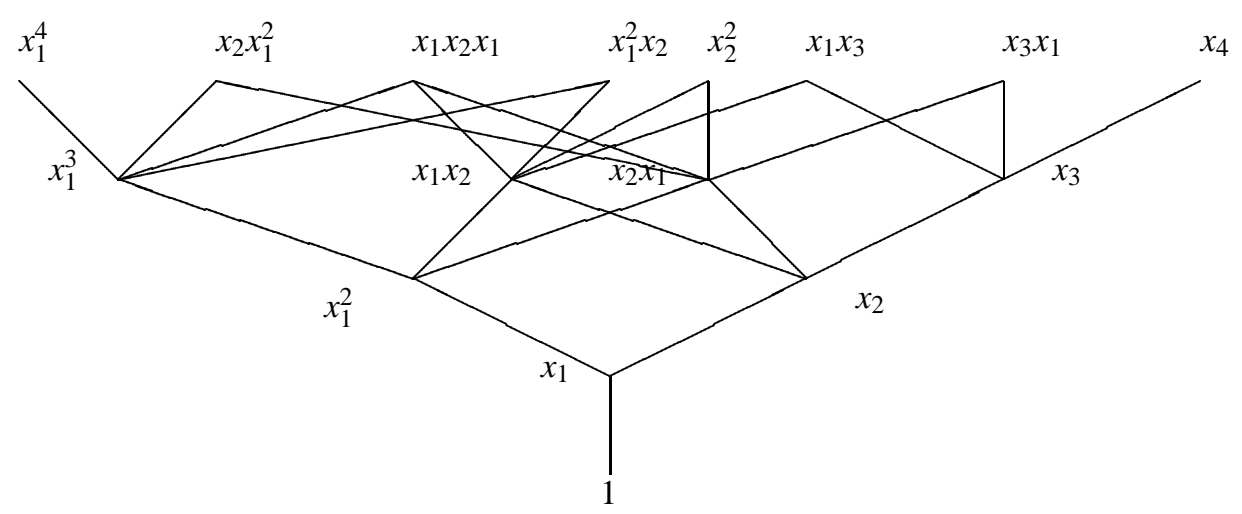

Fig. 3: The Hasse diagram of $\mathfrak{N}$.

Theorem 6.2. $\quad$ 1. $\mathfrak{C}$ is the intersection of all standard term orders on $[X]$.

2. $\mathfrak{C}_{n}$ is the intersection of all term orders on $\left[X_{n}\right]$.

3. The map $G \circ \log :([X], \mathfrak{C}) \rightarrow \mathbb{N}^{\omega}$ is an order-preserving monoid monomorphism, and $([X], \mathfrak{C})$ is isomorphic to the image, which we call $\mathcal{D}$. This image consists of all non-decreasing finitely supported sequences, and is order-isomorphic to the Young lattice of unordered number-partitions.

4. The image of $\left[X_{n}\right]$ correspond to the number-partitions whose diagrams have at most $n$ columns.

In other words, $G \circ \log$ is a $\omega$-multi-ranking, which happens to be an isomorphism onto its image. The relation between this poset and $\mathfrak{N}$ is as follows.

Theorem 6.3. With respect to the partial order $\mathfrak{N}$ on $X^{*}$, and the partial order $\mathfrak{C}$ on $[X], \sigma$ and $\sigma^{+}$are monotone. Furthermore, $\sigma^{+}(\sigma(p)) \nless p$, for all $p \in X^{*}$.

The same results hold for the restrictions of $\sigma$ and $\sigma^{+}$to $X_{n}^{*}$ and $\left[X_{n}\right]$.

Proof. If $X^{*} \ni m=x_{i_{1}} \cdots x_{i_{d}}$ then $\sigma(m)=x_{1}^{a_{1}} \cdots x_{N}^{a_{N}}$, with $a_{\ell}$ denoting the number of $j$ such that $x_{j}=\ell$. Clearly, $\sigma\left(m x_{1}\right)=x_{1} \sigma(m)$, which is $\geq \sigma(m)$ with respect to $\mathfrak{C}$. Furthermore,

$$
\begin{aligned}
\sigma\left(R_{j}(m)\right) & =\sigma\left(x_{i_{1}} \cdots x_{i_{j-1}} x_{i_{j}+1} x_{i_{j+1}} \cdots x_{i_{d}}\right) \\
& =x_{1}^{a_{1}} \cdots x_{j-1}^{a_{j-1}} x_{j}^{a_{j}-1} x_{j+1}^{a_{j+1}+1} x_{j+2}^{a_{j+2}} \cdots x_{N}^{a_{N}} \\
& =\frac{x_{j+1}}{x_{j}} m .
\end{aligned}
$$

By the definition of $\mathfrak{C}$, this is $\geq m$. 
Conversely, let $t=x_{1}^{a_{1}} \cdots x_{N}^{a_{N}} \in[X]$. Then $\sigma^{+}(t)=x_{1}^{a_{1}} \cdots x_{N}^{a_{N}} \in X^{*}$. It is clear that $\sigma^{+}\left(x_{1} t\right)=x_{1} \sigma^{+}(t) \geq$ $\sigma^{+}(t)$. Finally,

$$
\begin{aligned}
\sigma^{+}\left(\frac{x_{j+1}}{x_{j}} t\right) & =\sigma^{+}\left(x_{1}^{a_{1}} \cdots x_{j-1}^{a_{j-1}} x_{j}^{a_{j}-1} x_{j+1}^{a_{j+1}+1} x_{j+2}^{a_{j+2}} \cdots x_{N}^{a_{N}}\right) \\
& =x_{1}^{a_{1}} \cdots x_{j-1}^{a_{j-1}} x_{j}^{a_{j}-1} x_{j+1}^{a_{j+1}+1} x_{j+2}^{a_{j+2}} \cdots x_{N}^{a_{N}} \\
& =R_{a_{1}+a_{2}+\cdots+a_{j}}\left(\sigma^{+}(t)\right)
\end{aligned}
$$

So $\sigma^{+}$is isotone.

We recall (Lemma 2.2 on page 301) that $\sigma^{+}(\sigma(p))$ is the "sorted version" of $p \in X^{*}$. Hence, $p$ and $\sigma^{+}(\sigma(p))$ have the same multi-rank, and form an anti-chain.

The ranking $\Phi$ is the composition of $\sigma$ and the ranking of $\mathfrak{C}$, so the following diagram commutes:

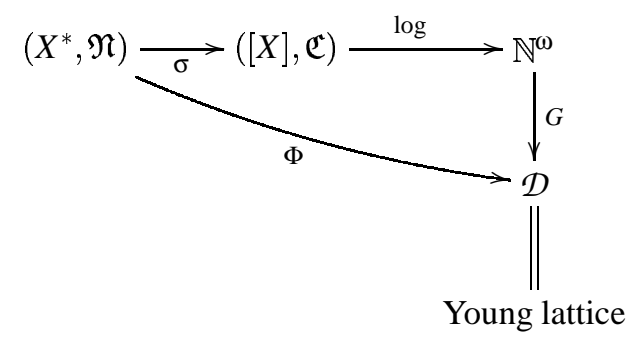

We can thus regard $\mathfrak{N}$ as a "non-commutative version" of the Young lattice. A illustrative interpretation is the following: we identify the Young lattice with $\mathcal{D}$, and $X^{*}, \mathfrak{N}$ with all the set of all lattice walks in the infinite-dimensional lattice $\mathbb{N}^{\omega}$, using the steps $\mathbf{f}_{1}=\mathbf{e}_{1}=(1,0,0,0,0 \ldots),, \mathbf{f}_{2}=\mathbf{e}_{1}+\mathbf{e}_{2}=(1,1,0,0,0 \ldots$,$) ,$ $\mathbf{f}_{3}=\mathbf{e}_{1}+\mathbf{e}_{2}+\mathbf{e}_{3}=(1,1,1,0,0, \ldots$,$) , et cetera. Then the multi-rank of such a walk is its endpoint, which$ lies in $\mathcal{D}$. When we restrict to 2 variables, the correspondence is easy to draw, as is shown in Figure 4 . It is easy to draw the effect of the "sorting" $\sigma^{+} \circ \sigma$, see Figure 5.

O
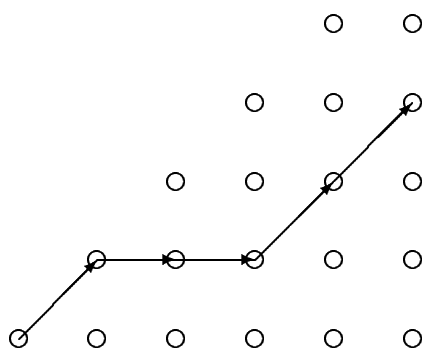

Fig. 4: The word $x_{2} x_{1}^{2} x_{2}^{2}$ as a path, bi-rank $(5,3)$
O
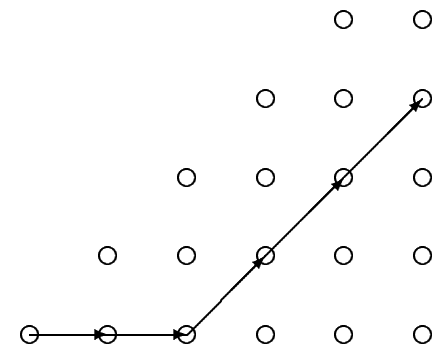

Fig. 5: $\sigma^{+}\left(\sigma\left(x_{2} x_{1}^{2} x_{2}^{2}\right)\right)=x_{1}^{2} x_{2}^{3}$ 


\section{Variants}

\section{1 "Sorted" term orders}

Recall that if $L, P$ are posets, then a Galois coconnection is a pair of maps $f: P \rightarrow L$ and $g: L \rightarrow P$ such that

1. $f$ and $g$ are order-preserving,

2. $g f(m) \geq m$ for all $m \in P$,

3. $f g(w) \leq w$ for all $w \in L$.

If we modify the partial order on $X^{*}$ slightly, $\left(\sigma, \sigma^{+}\right)$becomes a Galois coconnection.

Definition 7.1. Call a standard term order $>$ sorted if

$$
t x_{i} x_{j} s>t x_{j} x_{i} s \quad \text { for all } i<j, s, t \in X^{*}:
$$

Let $Q$ be the intersection of all sorted standard term orders on $X^{*}$, and for each positive integer $n, Q$ be the intersection of all sorted standard term orders on $X_{n}^{*}$.

Lemma 7.2. For any commutative monomial $m \in[X]$, the restriction of $Q$ to $\sigma^{-1}(m)$ (equivalently, to the set of monomials having multi-rank $G(\log (m)))$ is a chain.

Proof. Trivial.

Theorem 7.3. $m \leq m^{\prime}$ with respect to $Q$ if and only if $m^{\prime}$ can be obtained from $m$ by a sequence of applications of the following types:

(a) $t \mapsto x_{1} t$,

(b) $t \mapsto t x_{1}$,

(c) $t \mapsto R_{j}(t)$,

(d) $t x_{j} x_{i} s \mapsto t x_{i} x_{j} s$ for $i<j, s, t \in X^{*}$.

Furthermore, $\left(\sigma, \sigma^{+}\right)$is a Galois coconnection between $\left(X^{*}, Q\right)$ and $([X], \mathfrak{C})$.

If we restrict to $n$ variables, we get a Galois coconnection between $\left(X_{n}^{*}, Q_{n}\right)$ and $\left(\left[X_{n}\right], \mathfrak{C}\right)$.

Proof. The first assertion is similar to Theorem 3.5 on page 302; we omit the proof.

It remains to show that $\left(\sigma, \sigma^{+}\right)$is a Galois coconnection.

If $m^{\prime}$ is obtained from $m$ by a sequence of operations of type (a),(b), (c) then we know from Theorem 6.3 on page 308 that $\sigma\left(m^{\prime}\right) \geq \sigma(m)$. Furthermore, $\sigma\left(t x_{j} x_{i} s\right)=\sigma\left(t x_{i} x_{j} s\right)$. Hence, $\sigma$ is order preserving.

Since $Q \supset \mathfrak{N}$, it follows from Theorem 6.3 on page 308 that $\sigma^{+}$is order preserving.

For $m=x_{1}^{a_{1}} \ldots x_{\ell}^{a \ell}$ we have that $\sigma^{+} \sigma\left(x_{i_{1}} \ldots x_{i_{d}}\right)=x_{1}^{a_{1}} \ldots x_{\ell}^{a \ell}$, with $a_{j}$ denoting the number of $i$ such that $a_{i}=j$. In other word, it is the "sorted version" of $m$, and it can be obtained from $m$ by a sequence of operations of type (d): just perform a "bubble sort". Hence $\sigma^{+} \sigma(m) \geq m$ for all $m \in X^{*}$.

We know that $\sigma^{+}(w)=w$ for all $w \in[X]$, so in particular, $\sigma \sigma^{+}(w) \leq w$ for all $w \in[X]$.

We note that with respect to the Galois coconnection above, the closed elements in $\left(X^{*}, Q\right)$ are the "sorted" ones, and that all elements in $[X], \mathfrak{C}$ are coclosed. We show a part of the Hasse diagrams for $\left.\mathfrak{C}_{2}\right)$ and $Q_{2}$ in Figures 6 on the next page and 7 on the following page. 


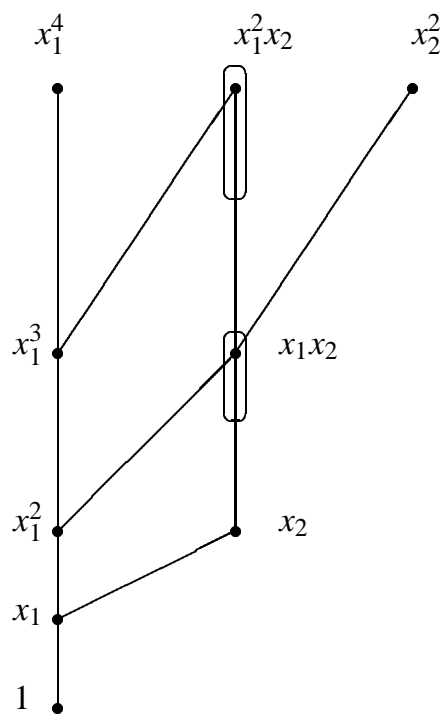

Fig. 6: The Hasse diagram of $\mathfrak{C}_{2}$

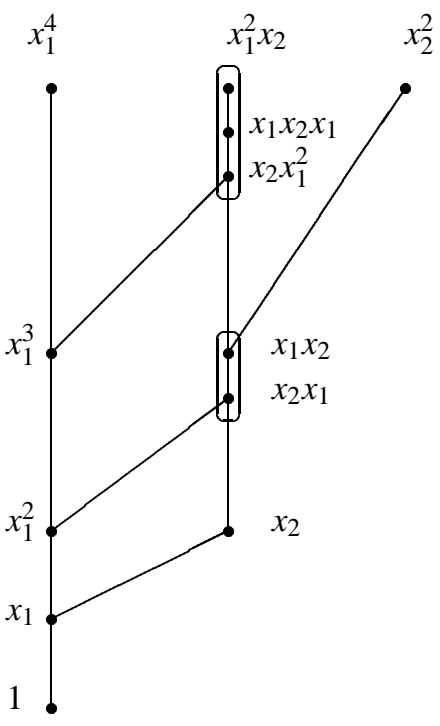

Fig. 7: The Hasse diagram of $Q_{2}$ is obtained from that of $\mathfrak{C}_{2}$ by replacing some elements by chains.

\subsection{Total degree term orders}

Theorem 7.4. Let $n, d$ be non-negative integers.

1. The restriction of $\mathfrak{N}$ to the set of words in $X^{*}$ of total degree d is isomorphic to $\mathbb{N}^{d}$,

2. The restriction of $\mathfrak{N}_{n}$ to the set of words in $X_{n}^{*}$ of total degree $d$ is isomorphic to the $d$-fold ordinal product of the chain with n elements,

3. In particular, the restriction of $\mathfrak{N}_{2}$ to the set of words in $X_{2}^{*}$ of total degree $d$, is isomorphic to the free boolean lattice on d elements.

Proof. The map

$$
X^{*} \ni x_{i_{1}} \cdots x_{i_{d}} \mapsto\left(i_{1}, \ldots, i_{d}\right) \in \mathbb{N}^{d}
$$

is an order-preserving bijection. If the word is in $X_{n}^{*}$ then $1 \leq i_{j} \leq n$.

Theorem 7.5. Let $P$ denote the partial order on $X^{*}$ which is the intersection of all degree-compatible standard term orders, where a term order is degree-compatible if $|m|>\left|m^{\prime}\right| \Longrightarrow m>m^{\prime}$. Then $P \subset \mathfrak{N}$, and $P$ is the ordinal sum

$$
\mathbb{N}^{0}+\mathbb{N}^{1}+\mathbb{N}^{2}+\cdots
$$

Similarly, if $P_{n}$ denotes the partial order on $\left\{x_{1}, \ldots, x_{n}\right\}^{*}$ which is the intersection of all degree-compatible standard term orders, then $P_{n} \subset \mathfrak{N}_{n}$, and $P_{n}$ is the ordinal sum

$$
C_{n}^{0}+C_{n}^{1}+C_{n}^{2}+\cdots
$$


where $C_{n}$ is the chain with $n$ elements. In particular, $P_{2}$ is the ordinal sum

$$
B_{0}+B_{1}+B_{2}+\cdots
$$

where $B_{i}$ is the free boolean lattice on i elements.

Proof. Follows from Theorem 7.4 on the page before.

\section{References}

[1] Martin Aigner. Combinatorial Theory, volume 234 of Grundlehren der mathematischen Wissenschaften. Springer, 1979.

[2] A. M. Bigatti. Upper bounds for the Betti numbers of a given Hilbert function. Communications in Algebra, 21:2317-2334, 1993.

[3] A. M. Bigatti. Aspetti Combinatorici e Computazionali dell'Algebra Commutativa. PhD thesis, Università di Torino, 1995.

[4] A. M. Bigatti and L. Robbiano. Borel sets and sectional matrices. Annals of Combinatorics, 1(3):197-213, 1997.

[5] David Eisenbud. Commutative Algebra with a View Toward Algebraic Geometry, volume 150 of Graduate Texts in Mathematics. Springer Verlag, 1995.

[6] S. Eliahou and M. Kervaire. Minimal resolutions of some monomial ideals. Journal of Algebra, 129:1-25, 1990.

[7] Mark Green and Michael Stillman. A tutorial on generic initial ideals. In Gröbner bases and applications (Linz, 1998), pages 90-108. Cambridge Univ. Press, Cambridge, 1998.

[8] Mark L. Green. Generic initial ideals. In Proceedings of the Summer School on Commutative Algebra, volume 2, pages 16-85, CRM, Barcelona, 1996.

[9] Graham Higman. Ordering by divisibility in abstract algebras. Proc. London Math. Soc. (3), 2:326336, 1952.

[10] Ursula Martin and Elizabeth Scott. The order types of termination orderings on monadic terms, strings and multisets. J. Symbolic Logic, 62(2):624-635, 1997.

[11] Keith Pardue. Nonstandard Borel-fixed ideals. PhD thesis, Brandeis University, 1994.

[12] Jan Snellman. On some partial orders associated to generic initial ideals. Séminaire Lotharingien de Combinatoire, B43h, 2000. 